\title{
Detection of Keratoconus With a New Biomechanical Index
}

\author{
Riccardo Vinciguerra, MD; Renato Ambrósio, Jr., MD, PhD; Ahmed Elsheikh, PhD; \\ Cynthia J. Roberts, PhD; Bernardo Lopes, MD; Emanuela Morenghi, PhD; Claudio Azzolini, MD; \\ Paolo Vinciguerra, MD
}

\section{ABSTRACT}

PURPOSE: To evaluate the ability of a new combined biomechanical index called the Corvis Biomechanical Index (CBI) based on corneal thickness profile and deformation parameters to separate normal from keratoconic patients.

METHODS: Six hundred fifty-eight patients (329 eyes in each database) were included in this multicenter retrospective study. Patients from two clinics located on different continents were selected to test the capability of the $\mathrm{CBI}$ to separate healthy and keratoconic eyes in more than one ethnic group using the Corvis ST (Oculus Optikgeräte $\mathrm{GmbH}$, Wetzlar, Germany). Logistic regression was employed to determine, based on Database 1 as the development dataset, the optimal combination of parameters to accurately separate normal from keratoconic eyes. The $\mathrm{CBI}$ was subsequently independently validated on Database 2.

RESULTS: The CBI included several dynamic corneal response parameters: deformation amplitude ratio at 1 and $2 \mathrm{~mm}$, applanation 1 velocity, standard deviation of deformation amplitude at highest concavity, Ambrósio's Relational Thickness to the horizontal profile, and a novel stiffness parameter. The receiver operating characteristic curve analysis of the training database showed an area under the curve of 0.983 . With a cut-off value of $0.5,98.2 \%$ of the cases were correctly classified with $100 \%$ specificity and $94.1 \%$ sensitivity. In the validation dataset, the same cut-off point correctly classified $98.8 \%$ of the cases with $98.4 \%$ specificity and $100 \%$ sensitivity.

CONCLUSIONS: The CBI was shown to be highly sensitive and specific to separate healthy from keratoconic eyes. The presence of an external validation dataset confirms this finding and suggests the possible use of the $\mathrm{CBI}$ in everyday clinical practice to aid in the diagnosis of keratoconus.

[J Refract Surg. 2016;32(12):803-810.]

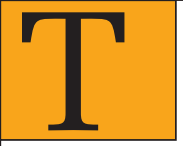

he early diagnosis of corneal ectasia is of foremost importance in both screening for refractive surgery and the early treatment of keratoconus. Topography or tomography analysis using either videokeratography or optical coherence tomography instruments can help detect alteration in the shape of the cornea such as thinning and increased curvature. However, these instruments cannot measure the mechanical stability, which is thought to be the initiating event of the disease, even before notable changes in corneal morphology take place., ${ }^{1,2}$ For this reason, there has been increasing interest in developing instruments to measure the in vivo biomechanical properties of the cornea to aid the diagnosis of an ectasia in a "biomechanical" stage, when topography and tomography are nor-

From the Department of Surgical Sciences, Division of Ophthalmology, University of Insubria, Varese, Italy ( $R V, C A)$; Rio de Janeiro Corneal Tomography and Biomechanics Study Group, Rio de Janeiro, Brazil (RA, $B L)$; the Department of Ophthalmology, Federal University of São Paulo, São Paulo, Brazil (RA, BL); the School of Engineering, University of Liverpool, Liverpool, United Kingdom (AE); NIHR Biomedical Research Centre for Ophthalmology, Moorfields Eye Hospital NHS Foundation Trust and UCL Institute of Ophthalmology, London, United Kingdom (AE); the Department of Ophthalmology \& Visual Science, Department of Biomedical Engineering, The Ohio State University, Columbus, Ohio (CJR); the Biostatistic Unit, Humanitas Research Hospital, Milan, Italy (EM); Eye Center, Humanitas Clinical and Research Center, Milan, Italy (PV); Humanitas University, Milan, Italy (PV); and Vincieye Clinic, Milan, Italy $(P V)$.

(C) 2016 Vinciguerra, Ambrosió, Elsheik, et al.; licensee SLACK Incorporated. This is an Open Access article distributed under the terms of the Creative Commons Attribution 4.0 International (https://creativecommons.org/licenses/by/4.0). This license allows users to copy and distribute, to remix, transform, and build upon the article, for any purpose, even commercially, provided the author is attributed and is not represented as endorsing the use made of the work.

Submitted: April 19, 2016; Accepted: June 23, 2016

Drs. Ambrósio, Vinciguerra, and Roberts are consultants for and Dr. Elsheikh has received research funding from Oculus Optikgeräte $\mathrm{GmbH}$. The remaining authors have no financial or proprietary interest in the materials presented herein.

Drs. Riccardo Vinciguerra and Paolo Vinciguerra contributed equally to this work and should be considered as equal first authors.

Correspondence: Paolo Vinciguerra, MD, Humanitas Clinical and Research Center, Via Manzoni 56, 20089 Rozzano, Milan, Italy. E-mail: paolo.vinciguerra@ humanitas.it

doi:10.3928/1081597X-20160629-01 
mal. The first one to be developed was the Ocular Response Analyzer (ORA) (Reichert Inc., Depew, NY). ${ }^{3}$ The ORA measures corneal behavior during a bidirectional applanation process induced by an air jet and produces estimates of corneal hysteresis and corneal resistance factor, along with a set of 38 waveformderived parameters. ${ }^{4-6}$ The capability of the ORA to diagnose keratoconus was tested in several articles ${ }^{6-8}$ but never reached the gold standard.

The Corvis ST (Oculus Optikgeräte GmbH, Wetzlar, Germany) was later introduced as a noncontact tonometer that monitors the response of the cornea to an air pressure pulse using an ultra-high speed Scheimpflug camera and uses the captured image sequence to produce estimates of intraocular pressure (IOP) and deformation response parameters. ${ }^{9}$ The Corvis ST does not currently provide an automatic analysis of corneal biomechanics.

The aim of this article was to develop a combined biomechanical index called the Corvis Biomechanical Index (CBI) based on different dynamic corneal response (DCR) parameters provided by the Corvis ST to separate keratoconic from normal eyes.

\section{PATIENTS AND METHODS}

Six hundred fifty-eight patients were included in this multicenter retrospective study. The patients from two clinics located in two different countries were selected to include variability from different continents and to test the capability of the CBI to separate healthy and keratoconic eyes in more than one ethnic group. A total of 329 patients (227 healthy and 102 keratoconic) were enrolled from the Rio de Janeiro Corneal Tomography and Biomechanics Study Group, Rio de Janeiro, Brazil (Database 1) and 329 patients (251 healthy and 78 keratoconic) from the Vincieye Clinic in Milan, Italy (Database 2). The institutional review board ruled that approval was not required for this record review study, and it was conducted according to the tenets of the Declaration of Helsinki. However, participants provided informed consent before using their data in the study.

All patients had a complete ophthalmic examination including the Corvis ST and Pentacam (Oculus Optikgeräte $\mathrm{GmbH}$ ) examinations.

The inclusion criterion of this study for the keratoconic population was the presence of bilateral clear keratoconus without any previous ocular procedures, such as corneal collagen cross-linking or intracorneal ring segment implantation. Conversely, the inclusion criteria for the healthy participants were the presence in the database of a Corvis ST examination, a Belin/Ambrósio Enhanced Ectasia total deviation index (BAD-D) score from the Pentacam with less than 1.6 standard deviations (SDs) from normative values in both eyes, and a signed informed consent. Exclusion criteria included any previous ocular surgery or disease, myopia greater than 10.00 diopters (D), and any concomitant or previous glaucoma or hypotonic therapies. The BAD-D cutoff of 1.6 SD was used because it is described as the best performing screening parameter with values of 1.65 and 1.88 associated, respectively, with $95 \%$ and $97.5 \%$ confidence intervals and an acceptable false-negative rate below $1 \% .{ }^{10}$ Moreover, to confirm the diagnosis of either keratoconus or normality, all examinations at the Vincieye Clinic were blindly reevaluated by Dr. Ambrósio to confirm the diagnosis. Similarly, all of the examinations at the Rio de Janeiro Clinic were blindly reevaluated by Dr. Paolo Vinciguerra.

Only Corvis ST examinations with good quality scores that enabled calculation of all deformation parameters were included in the analysis. The quality of the examination (quality score) was analyzed based on the following factors: model deviation of front and back surface, lost images, valid data points for corneal front and back surface, and alignment in $\mathrm{x}, \mathrm{y}$, and $\mathrm{z}$ position. In addition, the profile of the air pulse for each measurement was compared with the desired value.

Model deviations occurred if the edge detection and polynomial fit did not give reliable results (eg, if the edge detection is affected by eyelashes or eyelids).

Some of the 140 images might be lost if the patient blinks. If one or more images are missing, the parameter "lost images" indicates the poor quality of the examination. If only some data points are missing, the parameter "valid points" tests whether sufficient data points are available. For the anterior surface of the cornea, more than $95 \%$ of valid data points should be available for each image. The alignment position is also analyzed: the distance of the highest point to the corneal vertex in $x, y$ coordinates is measured. In addition, the deviation in $\mathrm{z}$ position to the desired measurement distance $(11 \mathrm{~mm})$ is analyzed. Finally, the deviation of the air pulse to the reference air pulse is calculated and should not exceed $5 \%$ deviation over the whole air pulse curve.

All measurements with the Corvis ST were taken by the same experienced technicians and captured by automatic release to ensure the absence of user dependency. Additionally, a second manual, frame-by-frame analysis of the examination, made by an independent masked examiner, was performed to ensure the quality of each acquisition. The main criterion was good edge detection over the whole deformation response, with the exclusion of alignment errors (x-direction). Similarly, blinking errors were omitted. 
The Corvis ST uses an ultra-high speed Scheimpflug camera that captures 4,330 images per second and covers $8 \mathrm{~mm}$ of the central cornea in a single horizontal meridian. The instrument's light source is an LED light of $455 \mathrm{~nm}$ wavelength. The instrument applies an air impulse with a maximum pressure of 25 kiloPascals. A quality score is available just after the measurement is taken for assessing the reliability of the measurement. This is based on a series of parameters that are obtained so that a quality score is also available for the pachymetry and IOP data. The Corvis ST output parameters from each measurement using research software were exported to a spreadsheet and analyzed.

Only one eye per patient was randomly included in the analysis to avoid the bias of the relationship between bilateral eyes that could influence the analysis result.

\section{DCR PARAMETERS}

Sixteen DCR parameters provided by Corvis ST were evaluated. These included: A1 velocity (speed of corneal apex at first applanation), A2 velocity (speed of corneal apex at second applanation), peak distance (distance between the two bending peaks created in the cornea at the maximum concavity state), highest concavity radius (radius of the central cornea at the maximum concavity state, based on a parabolic fit), and deformation amplitude (the largest displacement of corneal apex in the anterior-posterior direction at the moment of highest concavity). ${ }^{4,9}$ During the measurement, the whole eye movement, another measured parameter, affects deformation amplitude. As the cornea deforms and approaches maximum displacement, the whole eye displays a slow linear motion in the anterior-posterior direction. Another DCR is the deflection amplitude denoting displacement of corneal apex in reference to the cornea's initial state. Therefore, the deformation amplitude is the sum of corneal deflection amplitude and whole eye movement. Additionally, the deflection area describes the "displaced" area of the cornea in the analyzed horizontal sectional plane.

Other DCR parameters can be extrapolated from the highest concavity moment, including inverse concave radius and peak distance. The inverse concave radius $(1 / R)$ is plotted over the duration of the air pulse and the integrated sum is calculated between the first and second applanation events. ${ }^{4,9}$ The peak distance describes the distance between the two highest points of the cornea's temporal-nasal cross-section at the highest concavity moment. ${ }^{4}$

Two new parameters called central-peripheral deformation amplitude ratio (deformation amplitude ra- tio) measured at 1 or $2 \mathrm{~mm}$ and one additional DCR parameter named deflection amplitude ratio describe the ratio between the deformation/deflection amplitude at the apex and the average deformation/deflection amplitude measured at 1 or $2 \mathrm{~mm}$ from the center. The greater the deformation amplitude ratio and deflection amplitude ratio, the less resistant is the cornea to deformation. Therefore, one would expect higher values of deformation amplitude ratio and deflection amplitude ratio to be associated with softer corneas.

The Delta Arclength (HCdArclength) is another DCR parameter that describes the change in Arclength during the highest concavity moment from the initial state, in a defined $7-\mathrm{mm}$ zone. This parameter is calculated $3.5 \mathrm{~mm}$ from the apex to both sides in the horizontal direction. The temporal changes in the Delta Arclength are also calculated for the exact same zone and a plot is generated.

Further, and in addition to the central corneal thickness, the Corvis ST generates a new index called Ambrósio's Relational Thickness to the horizontal profile (ARTh), which is based on the thickness profile in the temporal-nasal direction ${ }^{11}$ as follows:

1. Corneal thickness is calculated at points with 0.2 $\mathrm{mm}$ spacing and the percentage thickness increase is calculated at each point relative to the smallest value.

2. The ratio between the percentage values (percentage thickness increases) and the corresponding normative values is calculated for each position along the complete thickness profile.

3. The average ratio for all positions provides the Pachymetric Progression Index: a value higher than 1 indicates a faster thickness increase than normal and a lower value indicates a slower thickness increase toward the periphery than normal.

4. The division between corneal thickness at the thinnest point and the Pachymetric Progression Index provides ARTh (ARTh = corneal thickness thinnest/pachymetric progression). A lower value indicates a thinner cornea and/or a faster thickness increase toward the periphery.

The force balance between the external air pressure and the IOP is determined at first applanation (A1), which defines the reference position for the development of a novel stiffness parameter (SP-A1) in the form of force divided by displacement. Therefore, the SP-A1 is defined as resultant pressure (Pr) divided by deflection amplitude at A1. Pr is defined as the adjusted pressure at A1 (adj AP1) minus a bio- 
mechanically corrected IOP value (bIOP), resulting in the following equation: SP-A1 = (adjusted AP1 bIOP) / A1 deflection amplitude.

The spatial and temporal profiles of the Corvis ST air pressure pulse were measured using hot wire anemometry, ${ }^{12}$ and adjusted AP1 was calculated as the air pressure impinging on the cornea at the time and position of applanation. The bIOP value was derived by finite element simulations that take into account the influence of central corneal thickness, age, and DCR parameters. This value has been validated both experimentally and clinically. ${ }^{13}$

The standard deviation (SD) of the deformation amplitude at highest concavity, relative to the correlation of deformation amplitude with bIOP in 705 eyes of 705 patients, ${ }^{14}$ is used to calculate the new parameter: SD-deformation amplitude. The SD value provides the multiple of SDs the measured value of highest concavity deformation amplitude is above the regression line between highest concavity deformation amplitude and bIOP in the referenced dataset. By taking this regression analysis into account, the confounding influence of IOP can be reduced.

\section{Statistical ANALYSis}

Receiver operating characteristic (ROC) curves were applied to determine the overall predictive accuracy of dynamic corneal response parameters and the combination of them, as described by the area under the curve (AUC). These curves are obtained by plotting sensitivity versus specificity, which is calculated for each value observed. An area of $100 \%$ implies that the test perfectly discriminates between groups.

Logistic regression with forward stepwise inclusion was employed to determine the optimal combination of best predictors from the individual indices for the creation of a CBI for the accurate separation between normal and keratoconic eyes, using Database 1 (Training dataset) for development. The parameters included in the analysis were bIOP, pachymetry (central corneal thickness), deformation amplitude, SD-deformation amplitude, applanation 1 velocity, peak distance, HCdArclength, highest concavity deflection area, deformation amplitude ratio $2 \mathrm{~mm}$, deformation amplitude ratio $1 \mathrm{~mm}$, deflection amplitude ratio, inverse concave radius, radius highest concavity, stiffness parameter-A1, and ARTh.

To make the coefficients of the logistic regression less dependent on extreme values, outliers for the CBI coefficients were excluded. After the refinement of the coefficients, outliers were re-included to test the capability of the CBI to separate normal from keratoconic eyes in the complete training dataset (Database 2). Optimal cut-off points of the CBI were obtained from the
ROC curves as those closest to the perfect classification point. Subsequently, to exclude overfitting, the parameter combination was independently validated using Database 2 (validation dataset).

The statistical analysis was performed with SPSS software (version 23; IBM Corporation, Armonk, NY).

\section{RESULTS}

Two hundred eighty-four left eyes and 374 right eyes were included. Database 1 included 152 left and 177 right eyes and Database 2 included 147 left and 182 right eyes. The mean age \pm SD of normal eyes was $37 \pm 12$ years in Database 1 and $32 \pm 12$ years in Database 2, whereas the mean age of keratoconic patients was $43 \pm 17$ years in Database 1 and $37 \pm 17$ years in Database 2. The study included 316 women and 342 men with a sex ratio (male to female) of 0.83 in normal patients and 2.27 in keratoconic patients. Dataset 1 comprised 169 women and 160 men with a sex ratio of 0.72 in normal patients and 1.76 in keratoconic patients. Dataset 2 included 147 women and 182 men, with a sex ratio of 0.95 in normal patients and 3.33 in keratoconic patients.

Considering only the keratoconic population in Database 1, the mean maximum keratometry (Kmax) value was $55.53 \pm 9.24 \mathrm{D}$ and the mean $\mathrm{BAD}$-D value was $9.98 \pm 17.23$. In Database 2, the mean Kmax value was $54.11 \pm 6.17 \mathrm{D}$ and the mean BAD-D value was $8.26 \pm 0.44$. None of these differences were statistically significant $(P>.05)$.

The Topographic Keratoconus Classification (TKC) provided by the Pentacam ${ }^{15}$ for both databases of the keratoconic patients is summarized in Table A (available in the online version of this article).

The stepwise logistic regression, based on Database 2 (training dataset), produced the following formula:

$$
\mathrm{CBI}=\operatorname{EXP}(\text { Beta }) /(1+\operatorname{EXP}(\text { Beta }))
$$

where Beta $=$ B1 * A1Velocity + B2 * ARTh + B3 * Stiffness Parameter-A1 + B4 * DARatio1mm + B5 * DARatio2mm + B6 * SD-Deflection Amplitude + B7

and $\mathrm{B} 1=-59.487, \mathrm{~B} 2=-0.027 ; \mathrm{B} 3=-0.092, \mathrm{~B} 4=-27.169$, $\mathrm{B} 5=5.472, \mathrm{~B} 6=-0.599, \mathrm{~B} 7=46.576$.

Values of all constants used in the equation were highly significant $(P<.01)$.

The ROC curve analysis of the training dataset (Database 1) showed an AUC of 0.983 (Figure 1A). With a cut-off value of $0.5,98.2 \%$ of the cases were correctly classified with $100 \%$ specificity and $94.1 \%$ sensitivity. Table 1 shows the gain in sensitivity and specificity 

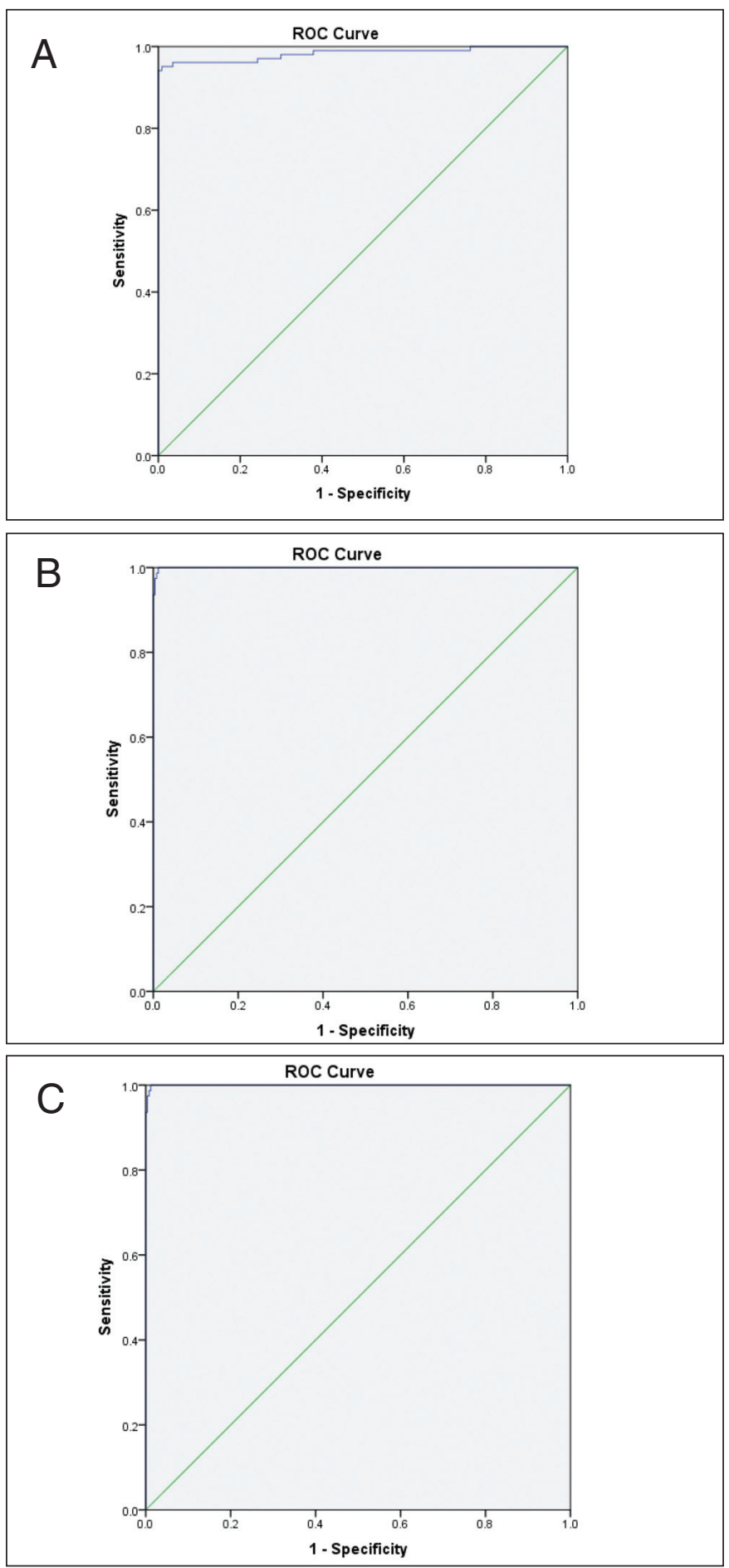

Figure 1. The receiver operating characteristic (ROC) of (A) the training dataset (1) with an area under the curve of 0.983 , (B) the validation dataset (2) with an area under the curve of 0.999 , and (C) the combined dataset with an area under the curve of 0.990 .

with each step of the logistic regression to create the CBI.

In the validation dataset (Database 2), the AUC was 0.999 and the same cut-off point correctly classified

\begin{tabular}{|c|c|c|c|}
\hline \multicolumn{4}{|c|}{$\begin{array}{c}\text { TABLE } 1 \\
\text { Gain in Sensitivity and Specificity With } \\
\text { Each Step of the Logistic Regression } \\
\text { to Create the CBI }\end{array}$} \\
\hline Step & Disease 0 & Disease 1 & $\%$ Correct \\
\hline \multicolumn{4}{|l|}{1} \\
\hline Disease 0 & 221 & 6 & 97.4 \\
\hline Disease 1 & 14 & 88 & 86.3 \\
\hline Overall \% & - & - & 93.9 \\
\hline \multicolumn{4}{|l|}{2} \\
\hline Disease 0 & 222 & 5 & 97.8 \\
\hline Disease 1 & 11 & 91 & 89.2 \\
\hline Overall \% & - & - & 95.1 \\
\hline \multicolumn{4}{|l|}{3} \\
\hline Disease 0 & 222 & 5 & 97.8 \\
\hline Disease 1 & 8 & 94 & 92.2 \\
\hline Overall \% & - & - & 96.0 \\
\hline \multicolumn{4}{|l|}{4} \\
\hline Disease 0 & 224 & 3 & 98.7 \\
\hline Disease 1 & 9 & 93 & 91.2 \\
\hline Overall \% & - & - & 96.4 \\
\hline \multicolumn{4}{|l|}{5} \\
\hline Disease 0 & 226 & 1 & 99.6 \\
\hline Disease 1 & 7 & 95 & 93.1 \\
\hline Overall \% & - & - & 97.6 \\
\hline \multicolumn{4}{|l|}{6} \\
\hline Disease 0 & 227 & 0 & 100 \\
\hline Disease 1 & 6 & 96 & 94.1 \\
\hline Overall \% & - & - & 98.2 \\
\hline \multicolumn{4}{|c|}{$\begin{array}{l}\text { CBI = Conis Biomechanical Index; Step } 1=\text { Ambrósio's Relational } \\
\text { Thickness to the horizontal profile; Step } 2=\text { stiffness parameter at appla- } \\
\text { nation } 1 \text { (A1); Step } 3=\text { A1 velocity; Step } 4 \text { = deformation amplitude ratio } \\
2 \mathrm{~mm} \text {; Step } 5=\text { deformation amplitude ratio; Step } 6=\text { standard deviation } \\
\text { of deformation amplitude }\end{array}$} \\
\hline
\end{tabular}

$98.8 \%$ of the cases with $98.4 \%$ specificity and $100 \%$ sensitivity (Figure 1B). The ROC curve analysis of the combined datasets showed an AUC of 0.990 and a good predictive accuracy of the CBI (Figure 1C).

Figure 2 shows the comparison of the ROC curves of the single parameters and the CBI.

\section{DISCUSSION}

There is a strong body of literature supporting the observation that keratoconic corneas are significantly "softer" or have lower tangent modulus values than normal corneas. ${ }^{16,17}$ These observations led to a hypothesis on biomechanical pathogenesis of keratoconus based on existing biomechanical models and 


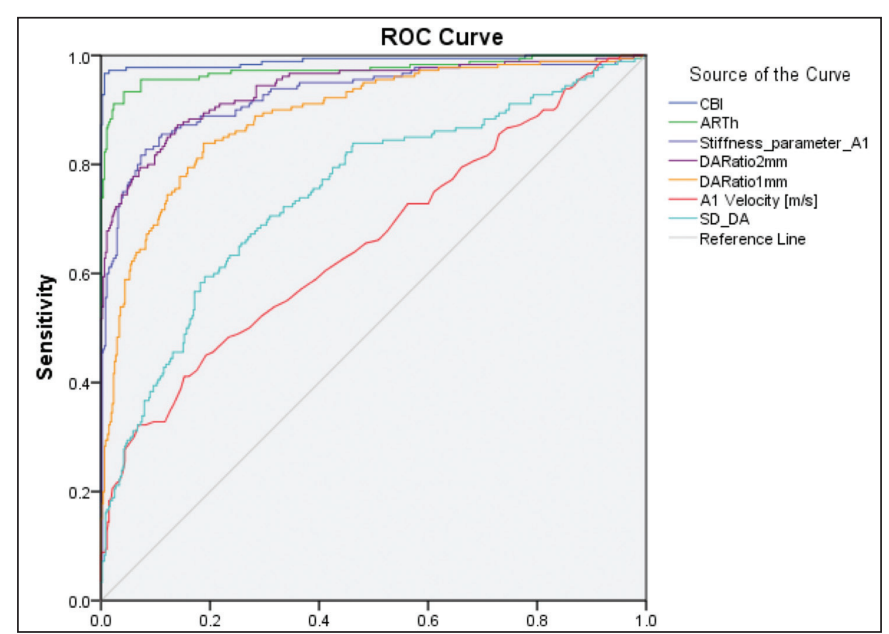

Figure 2. The comparison of the receiver operating characteristic (ROC) curves of the single parameters included in the Corvis Biomechanical Index (CBI): A1 velocity, deformation amplitude (DA) ratio 1 and $2 \mathrm{~mm}$, stiffness parameter $A 1$, standard deviation (SD) of DA at highest concavity, Ambrósio's Relational Thickness to the horizontal profile (ARTh), and CBI.

clinical topographic and tomographic data. ${ }^{1,18}$ The hypothesis, later supported by the studies of Scarcelli et al., ${ }^{2}$ proposed that the initiating event in keratoconus was a focal reduction in biomechanical properties resulting in tissue thinning as the softer area strains more than the surrounding stiffer areas. The cause may be an underlying pathology or perhaps a genetic predisposition with an external insult acting as a trigger, such as eye rubbing in a focal region. The result is that the focal reduction in tangent modulus generates, over time, greater deformation under IOP, causing focal thinning with increased stress and subsequent corneal bulging with loss of clear vision. The increase in curvature is associated with focal stress reduction as a compensatory mechanism, which contributes to an overall stress redistribution. This leads to a cycle of biomechanical decompensation driven by the disparity in corneal properties, with continued thinning and bulging, both of which redistribute the overall stress in the cornea. A direct consequence of this theory is that it might be possible to diagnose an ectasia based on observed deterioration in mechanical properties before the resulting changes in thickness and curvature profiles become evident. For this reason, the in vivo evaluation of corneal biomechanics is being developed and exploited in this study to achieve better outcomes for the early diagnosis of keratoconus. Given this aim, DCR parameters provided by the Corvis ST were analyzed and combined to create a biomechanical index aimed to separate normal from keratoconic patients.

This multicenter study included more than 600 cases from two different continents. The inclusion of only one eye per patient eliminated the risk of bias due to the relationship between bilateral eyes. Both datasets included only eyes with clear keratoconus and clearly normal eyes, but there are few minor differences. Considering the population of the two datasets, Database 1 had a slightly higher amount of early keratoconus cases; in particular, 6 cases were classified as normal by the TKC but abnormal with the BAD-D and the review by two experts (PV and RA). For this reason, Database 1 was used to create the formula because it was thought to be more challenging.

We recently published a study that included more than 700 healthy subjects that evaluated the DCR parameters mostly correlated with biomechanics and less affected by IOP and provided normality values for each of them. ${ }^{14}$ This background was critical to understand which parameter is more helpful in separating normal corneas from ectatic corneas.

Following the determination of the optimum parameter combination of the CBI, its diagnostic capability in distinguishing between normal and keratoconic eyes was assessed. The multivariate diagnostic model created showed high sensitivity and specificity with an overall AUC of 0.990. The CBI correctly classified more than $98 \%$ of the cases in both datasets.

To our knowledge, this is the first time in the literature that a combination of Corvis ST parameters is able to provide such efficiency for detecting keratoconus. Most earlier studies produced an AUC lower than $0.900,{ }^{19-21}$ even though some of these studies refer to subclinical cases. ${ }^{22}$

However, the dynamic Scheimpflug Corvis ST device is relatively new. Many similar studies were performed with the ORA, even if the device is not currently commercialized to diagnose keratoconus. However, the majority of the studies, even where the waveform derivatives were evaluated (37 additional parameters), produced a lower AUC. ${ }^{7,8,23-25}$ Hallahan et al. ${ }^{6}$ and Ventura et al. ${ }^{26}$ showed comparable AUC, but those studies included fewer cases and controls and did not have an external validation.

The presence of an external validation is of foremost importance when considering a multivariate analysis to exclude overfitting and because the cut-off value in one database may not produce the same results in a second independent one. The inclusion of this validation in our study, which produced even better results than with the training dataset, confirms the diagnostic performance of the CBI. To our knowledge, this was the first time in the literature that a larger validation dataset was used to confirm the diagnostic ability of an ectasia detection formula.

Additionally, it is important to comment that, even if the training dataset of the CBI reached a high sensi- 
tivity and specificity, the same cut-off value in the validation dataset produced even better results. This last finding excludes the risk of overfitting and confirms the diagnostic capability of the CBI for keratoconus.

A possible criticism of our study could be the decision to use ARTh and A1 velocity, the first because it is already a pure thickness profile with a good AUC, and the second because its AUC is not as good as the other parameters included. However, as shown in Table 1, sensitivity and specificity increased meaningfully with the addition of the other five DCR parameters, which confirms the importance of biomechanics in evaluating ectasia. Furthermore, ARTh can be considered either a thickness parameter inside the multivariate analysis to separate normal from keratoconic eyes or a correction parameter for the possible difference in thickness between the patients to correctly evaluate biomechanics. It is known that many DCR parameters are correlated with thickness. ${ }^{14,27,28}$ Regarding the inclusion of A1 velocity, even though it has a poor single capability of separating healthy from keratoconic eyes, its presence increased the sensitivity by two percentage points (see step 3 of Table 1). We hypothesize that, given its correlation with IOP, ${ }^{14}$ it compensates for the difference in IOP in the single cases. Instead of A1 velocity, one could also use IOP, but A1 velocity worked better in the combination. Furthermore, its beta value was highly significant.

It might be interesting to evaluate diurnal variations for keratoconus DCR parameters and how this might affect the measurements in healthy and keratoconic eyes. A study is already in progress to evaluate this possible effect, together with repeatability and reproducibility of DCR parameters of the Corvis ST.

In this study, we decided to exclude forme fruste keratoconus and subclinical cases from the databases to create the CBI. However, another study is in process, with promising results, to test the capability of the CBI alone and in combination with Pentacam indices to separate healthy eyes from eyes with forme fruste keratoconus.

Our study introduces the CBI for keratoconus diagnosis, which was shown to be highly sensitive and specific alone to separate healthy from ectatic eyes. The presence of an external validation dataset from another continent confirms this finding and suggests the possible use of the CBI in everyday clinical practice, together with topography and tomography, to aid the diagnosis of ectasia. More studies are in progress to show the capability of the CBI alone and in combination with tomographic indexes to separate healthy from subclinical cases.

\section{AUTHOR CONTRIBUTIONS}

Study concept and design ( $R V, R A, P V)$; data collection $(R V, B L)$; analysis and interpretation of data (RV, RA, AE, CJR, EM, $C A, P V)$; writing the manuscript ( $R V$ ); critical revision of the manuscript ( $R V$, $R A, A E, C J R, B L, M E, C A, P V)$; statistical expertise (RA, CJR, EM); supervision $(P V)$

\section{REFERENCES}

1. Ambrósio R Jr, Dawson DG, Salomão M, Guerra FP, Caiado AL, Roberts CJ. Biomechanics in keratoconus. In: Barbara A, ed. Textbook of Keratoconus: New Insights, 1st ed. New Delhi: Jaypee Brothers Medical Publishers; 2012:29-32.

2. Scarcelli G, Besner S, Pineda R, Yun SH. Biomechanical characterization of keratoconus corneas ex vivo with Brillouin microscopy. Invest Ophthalmol Vis Sci. 2014;55:4490-4495.

3. Luce DA. Determining in vivo biomechanical properties of the cornea with an ocular response analyzer. J Cataract Refract Surg. 2005;31:156-162.

4. Roberts CJ. Concepts and misconceptions in corneal biomechanics. J Cataract Refract Surg. 2014;40:862-869.

5. Mikielewicz M, Kotliar K, Barraquer RI, Michael R. Air-pulse corneal applanation signal curve parameters for the characterisation of keratoconus. Br J Ophthalmol. 2011;95:793-798.

6. Hallahan KM, Sinha Roy A, Ambrosio R Jr, Salomao M, Dupps WJ Jr. Discriminant value of custom Ocular Response Analyzer waveform derivatives in keratoconus. Ophthalmology. 2014;121:459-468

7. Galletti JG, Pfortner T, Bonthoux FF. Improved keratoconus detection by Ocular Response Analyzer testing after consideration of corneal thickness as a confounding factor. J Refract Surg. 2012;28:202-208.

8. Touboul D, Bénard A, Mahmoud AM, Gallois A, Colin J, Roberts CJ. Early biomechanical keratoconus pattern measured with an ocular response analyzer: curve analysis. J Cataract Refract Surg. 2011;37:2144-2150.

9. Ambrósio R Jr, Ramos I, Luz A, et al. Dynamic ultra high speed Scheimpflug imaging for assessing corneal biomechanical properties. Rev Bras Oftalmol. 2013;72:99-102.

10. Villavicencio GF, Henriquez MA, Izquierdo L Jr, Ambrosio RR Jr, Belin MW. Independent population validation of the Belin/ Ambrosio enhanced ectasia display: implications for keratoconus studies and screening. Int J Kerat Ect Cor Dis. 2014;3:1-8.

11. Lopes BT, Ramos IDC, Salomão MQ, Canedo ALC, Ambrósio R Jr. Perfil paquimétrico horizontal para a detecção do ceratocone. Rev Bras Oftalmol. 2015;74:382-385.

12. Roberts CJ, Mahmoud AM, Bons J, et al. New stiffness parameter in air puff induced corneal deformation analysis. Invest Ophth Vis Sci. 2016;57:ARVO E-Abstract 2390.

13. Joda AA, Shervin MMS, Kook D, Elsheikh A. Development and validation of a correction equation for Corvis tonometry. Comput Methods Biomech Biomed Engin. 2015;19:943-953.

14. Vinciguerra R, Elsheikh A, Roberts CJ, et al. Influence of pachymetry and intraocular pressure on dynamic corneal response parameters in healthy patients. J Refract Surg. 2016;32:550-561.

15. Goebels S, Eppig T, Wagenpfeil S, Cayless A, Seitz B, Langenbucher A. Staging of keratoconus indices regarding tomography, topography, and biomechanical measurements. Am J Ophthalmol. 2015;159:733-738.

16. Andreassen TT, Simonsen AH, Oxlund H. Biomechanical properties of keratoconus and normal corneas. Exp Eye Res. 1980;31:435-441. 
17. Anderson K, El-Sheikh A, Newson T. Application of structural analysis to the mechanical behaviour of the cornea. J R Soc Interface. 2004;1:3-15

18. Roberts CJ, Dupps WJ Jr. Biomechanics of corneal ectasia and biomechanical treatments. J Cataract Refract Surg. 2014;40:991998.

19. Ali NQ, Patel DV, McGhee CN. Biomechanical responses of healthy and keratoconic corneas measured using a noncontact scheimpflug-based tonometer. Invest Ophthalmol Vis Sci. 2014;55:3651-3659.

20. Steinberg J, Katz T, Lucke K, Frings A, Druchkiv V, Linke SJ. Screening for keratoconus with new dynamic biomechanical in vivo Scheimpflug analyses. Cornea. 2015;34:1404-1412.

21. Tian L, Huang YF, Wang LQ, et al. Corneal biomechanical assessment using corneal visualization Scheimpflug technology in keratoconic and normal eyes. J Ophthalmol. 2014;2014:147516.

22. Peña-García P, Peris-Martínez C, Abbouda A, Ruiz-Moreno JM. Detection of subclinical keratoconus through non-contact tonometry and the use of discriminant biomechanical functions. J Biomech. 2016;49:353-363.

23. Luz A, Lopes B, Hallahan KM, et al. Discriminant value of cus- tom ocular response analyzer waveform derivatives in forme fruste keratoconus. Am J Ophthalmol. 2016;164:14-21.

24. Ruiseñor Vázquez PR, Delrivo M, Bonthoux FF, Pförtner T, Galletti JG. Combining ocular response analyzer metrics for corneal biomechanical diagnosis. J Refract Surg. 2013;29:596-602.

25. Galletti JD, Ruiseñor Vázquez, Fuentes Bonthoux F, Pförtner T, Galletti JG. Multivariate analysis of the ocular response analyzer's corneal deformation response curve for early keratoconus detection. J Ophthalmol. 2015;2015:496382.

26. Ventura BV, Machado AP, Ambrósio R Jr, et al. Analysis of waveform-derived ORA parameters in early forms of keratoconus and normal corneas. J Refract Surg. 2013;29:637-643.

27. Huseynova T, Waring GO 4th, Roberts C, Krueger RR, Tomita M. Corneal biomechanics as a function of intraocular pressure and pachymetry by dynamic infrared signal and Scheimpflug imaging analysis in normal eyes. Am J Ophthalmol. 2014;157:885893.

28. Liu J, Roberts CJ. Influence of corneal biomechanical properties on intraocular pressure measurement: quantitative analysis. $J$ Cataract Refract Surg. 2005;31:146-155. 


\begin{tabular}{|c|c|c|c|}
\hline \multicolumn{4}{|c|}{$\begin{array}{c}\text { TABLE A } \\
\text { Topographic Keratoconus } \\
\text { Classiffication Provided by Pentacam }\end{array}$} \\
\hline Classification & Frequency & $\%$ & Cumulative \% \\
\hline \multicolumn{4}{|l|}{ Database 1} \\
\hline- & 6 & 5.9 & 5.9 \\
\hline 1 & 14 & 13.7 & 19.6 \\
\hline 1 to 2 & 14 & 13.7 & 33.3 \\
\hline 2 & 26 & 25.5 & 58.8 \\
\hline 2 to 3 & 8 & 7.8 & 66.7 \\
\hline 3 & 16 & 15.7 & 82.4 \\
\hline 3 to 4 & 8 & 7.8 & 90.2 \\
\hline 3 PMD & 2 & 2.0 & 92.2 \\
\hline 4 & 3 & 2.9 & 95.1 \\
\hline Abnormal & 2 & 2.0 & 97.1 \\
\hline Possible & 3 & 2.9 & 100 \\
\hline Total & 102 & 100 & - \\
\hline \multicolumn{4}{|l|}{ Database 2} \\
\hline 1 & 7 & 8.8 & 8.8 \\
\hline 1 to 2 & 12 & 15.0 & 23.8 \\
\hline 2 & 24 & 30.8 & 55.0 \\
\hline 2 to 3 & 13 & 16.3 & 71.3 \\
\hline 3 & 12 & 16.3 & 87.5 \\
\hline 3 to 4 & 7 & 8.8 & 96.3 \\
\hline 4 & 1 & 1.3 & 97.5 \\
\hline Possible & 2 & 2.5 & 100 \\
\hline Total & 78 & 100 & - \\
\hline \multicolumn{4}{|c|}{$\begin{array}{l}P M D=\text { pellucid marginal degeneration } \\
\text { The Pentacam is manufactured by Oculus Optikgeräte } \mathrm{GmbH} \text {, Wetzlar, } \\
\text { Germany. }\end{array}$} \\
\hline
\end{tabular}

